\title{
UNESTERIFIED FATTY ACID IN HUMAN BLOOD PLASMA. II. THE TRANSPORT FUNCTION OF UNESTERIFIED FATTY ACID
}

\author{
By ROBERT S. GORDON, JR. With the ASSISTANCE of AMELIA CHERKES AND \\ HERBERT GATES \\ (From the Laboratory of Cellular Physiology and Metabolism, National Heart Institute, \\ Bethesda, Md.)
}

(Submitted for publication October 18, 1956; accepted February 11, 1957)

In a recent publication (1), studies of the concentration of unesterified fatty acid (UFA) in the blood of normal human subjects were reported. The relation of the concentration of unesterified fatty acid to the nutritional state of the subject, together with the observation of arteriovenous differences in unesterified fatty acid concentration, formed the basis for the belief that this lipid fraction constitutes a transport form of fatty acid which can account for the movement of fats from adipose tissue depots into tissues requiring fat as an energy-yielding substrate. Other studies were reported which served to indicate the existence of hormonal mechanisms controlling the concentration of circulating unesterified fatty acid. At the same time, there appeared another publication (2), representing independent investigations with a different analytical method, in which virtually identical observations were reported (with the exception that no studies of arteriovenous differences were included). In view of the potentially great physiologic significance of plasma unesterified fatty acid, it was decided that the above-mentioned studies should be confirmed and extended, and that another analytical procedure, more accurate and convenient than that used earlier, should be developed. The method employed by Dole (2), though extremely convenient and rapid, did not seem suitable for the investigation of arteriovenous differences, since lactate interferes appreciably with the results obtained by that method. The data reported in the present publication were obtained by a procedure which has the advantage of high specificity, although it is appreciably more laborious than the method of Dole.

\section{MATERIALS AND METHODS}

Two groups of hospitalized patients were utilized as subjects for this study. The observations on arteriovenous differences were made on patients undergoing cardiac catheterization for the diagnosis of organic heart disease. These subjects were not strictly normal, but they had all received medical treatment as indicated to alleviate their symptoms, and none was used for this work who had clinically demonstrable congestive failure or suffered from any intercurrent metabolic disorder. They were fasting when studied, and were at rest physically, though for the most part there was considerable apprehension concerning the procedure to be carried out. The observations involving sequential samples of blood from the antecubital vein were made on normal conscientious objectors. These young men were hospitalized, had had a complete medical investigation, and were free of disease. They were fasting when studied, at rest physically, and, in general, were not greatly concerned emotionally with the procedure. In the observations involving sequential sampling of venous blood, care was taken to obtain all samples of a series from the same point in the same vein, in order to avoid superposing on the time course of the fatty acid concentration variations related to the fact that different veins might drain tissues whose behavior in extracting unesterified fatty acid from, or adding it to, blood might not be identical. Ideally, such studies should be done on arterial blood samples, but the advantages of arterial sampling were felt not to justify the discomfort involved.

Blood for analysis was collected in Wintrobe's oxalate mixture, refrigerated promptly, and centrifuged in the cold. An aliquot of plasma ( 1 to $3 \mathrm{ml}$., depending on the expected UFA concentration) was lyophilized in a $40-\mathrm{ml}$. conical glass-stoppered centrifuge tube, and the dry powder extracted for 30 minutes or more with exactly $25 \mathrm{ml}$. of a mixture of equal volumes of glacial acetic acid (Reagent Grade) and isoöctane (2,2,4 trimethyl pentane, Pure Grade, 99 per cent mol fraction minimum, obtained from Phillips Petroleum Co., Bartlesville, Oklahoma). Neither solvent required redistillation or other purification prior to use. The mixture was most conveniently delivered into the extraction vessels with a Krogh pipette. The tubes were then centrifuged, and the solvent decanted into a $60-\mathrm{ml}$. separatory funnel containing $2.5 \mathrm{ml}$. of 0.05 per cent sulfuric acid. After being shaken thoroughly, the funnel was centrifuged at approximately $100 \mathrm{G}$ for 5 minutes to effect complete separation of the two phases. The lower phase was discarded, and the upper octane phase washed twice with 25 $\mathrm{ml}$. of the same dilute sulfuric acid mixture, with recentrifugation after each washing and again after the second washing had been drained from the funnel. Ordinary 
glass separatory funnels with the stems cut off at $1 \mathrm{~cm}$. have proven satisfactory. The stopcocks may be greased with a product whose acid-base equivalence is negligible; for this purpose Lubriseal stopcock grease has been employed. The stopcock cores may be held firmly in place by a rubber band looped from the body of the funnel, over the handle of the stopcock core, and back to the stem. With these precautions, the funnels withstand gentle centrifugation without leakage, provided there is always an aqueous layer between the octane and the stopcock. After completion of the washing steps, two $5-\mathrm{ml}$. aliquots of the octane solution were pipetted into 12-ml. conical glass centrifuge tubes, $1 \mathrm{ml}$. of Nile Blue indicator solution was added, and the latter titrated to a pink end point with 0.02 normal aqueous sodium hydroxide solution delivered from a suitable microburette. Nitrogen gas served simultaneously to mix the two phases and to flush away atmospheric carbon dioxide. The Nile Blue indicator solution was freshly prepared by mixing nine parts of absolute ethanol and one part of a stock aqueous solution of commercial Nile Blue $A$ (0.02 per cent in water, washed with octane until free of extractable colored impurities). The titration was performed in duplicate on every extract, and the results averaged. After deduction of a reagent blank, this figure was used to calculate a single UFA concentration. Where duplicate values are reported, two identical plasma aliquots were lyophilized and four titrations carried out.

Statistical analysis of over one hundred plasmas analyzed in duplicate for unesterified fatty acid content indicated the standard error for a single determination to be $0.02 \mathrm{mEq}$. per L. Recovery experiments with stearic, palmitic, and oleic acids 1 added to plasma in the concentration of $1 \mathrm{mEq}$. per $\mathrm{L}$. indicated 96 to 102 per cent recovery. Many carboxylic acids, anticoagulants, and other substances were added to plasma in vitro without producing an appreciable difference in the measured UFA concentration, whereas a recovery of 0.2 per cent of most of the added materials should have been noticeable. The substances so tested included acetoacetate, beta hydroxybutyrate, succinate, pyruvate, lactate, urate, cholate, desoxycholate, oxalate, ethylenediaminetetraacetate, heparin, and protamine. Only in the case of lithocholate was a small recovery of the added material observed, amounting to 3 per cent of the lithocholate introduced. A number of the washed octane extracts have been tested for the presence of lipid phosphorus; the average concentration found amounted to $0.003 \mathrm{mg}$. per $\mathrm{ml}$. of plasma extracted. This amount of phospholipid is felt to be too small to introduce an appreciable error into the titration.

The analyses were for the most part begun within four hours after the withdrawal of the samples. When necessary, however, samples have been stored for periods ranging from overnight to several weeks prior to analysis. Experiment has shown that the samples may be stored without measurable increase in the UFA concentration at any one of three stages of the process, either as frozen

1 These acids were obtained from the Hormel Institute, Austin, Minnesota.
TABLE I

$A-V$ differences in $U F A$ concentration from coronary sinus (C.S.) and hepatic vein (H.V.) samples*

\begin{tabular}{|c|c|c|c|c|c|}
\hline \multirow[b]{2}{*}{ Patient } & \multirow{2}{*}{$\underset{\text { Venous }}{\text { site }}$} & \multicolumn{3}{|c|}{ UFA $(m E q . / L)}$. & \multirow{2}{*}{$\begin{array}{c}\mathrm{O}_{2} \\
\text { extraction } \\
\text { ratio } \\
\%\end{array}$} \\
\hline & & Arterial & Venous & Diff. & \\
\hline $\begin{array}{l}\text { M. C. } \\
\text { K. M. } \\
\text { F. E. } \\
\text { G. P. } \\
\text { L. McQ. } \\
\text { N. C. } \\
\text { F. C. } \\
\text { A. P. } \\
\text { M. Q. } \\
\text { J. S. }\end{array}$ & $\begin{array}{l}\text { C.S. } \\
\text { C.S. } \\
\text { C.S. } \\
\text { C. S. } \\
\text { C.S. } \\
\text { H. V. } \\
\text { H. V. } \\
\text { H. V. } \\
\text { H. V. } \\
\text { H. V. }\end{array}$ & $\begin{array}{r}.96 \\
.77 \\
1.04 \\
.37 \\
.82 \\
.29 \\
.94 \\
1.02 \\
1.03 \\
.59\end{array}$ & $\begin{array}{l}.64 \\
.53 \\
.73 \\
.21 \\
.71 \\
.24 \\
.74 \\
.73 \\
.74 \\
.42\end{array}$ & $\begin{array}{l}.32 \\
.24 \\
.31 \\
.16 \\
.11 \\
.05 \\
.20 \\
.29 \\
.29 \\
.17\end{array}$ & $\begin{array}{r}87 \\
70 \\
70 \\
38 \\
36 \\
31 \\
103\end{array}$ \\
\hline
\end{tabular}

* The figures reported are means of duplicate analyses. Oxygen extraction ratio is obtained by dividing the amount of oxygen required for the complete combustion of the extracted UFA by the total oxygen extraction measured on the same blood samples. In the calculation of oxygen required, it is assumed that the fatty acids are of average molecular weight 275 , and that no extractable UFA is carried in the erythrocytes.

plasma at $-20^{\circ} \mathrm{C}$, after lyophilization and addition of the extracting mixture (stored stoppered at $4^{\circ} \mathrm{C}$. to prevent evaporation of the organic solvents), or after the extracts have been washed and aliquots pipetted out for titration. Only deep-frozen plasma has been stored for more than 5 days. The result of the UFA determination on a plasma sample stored in the liquid state at $4^{\circ} \mathrm{C}$. or as lyophilized powder exposed to air was found not to be reliable.

\section{OBSERVATIONS}

To the data reported previously (1), there are now to be added a considerable number of new determinations of arteriovenous differences. The extraction of UFA from the blood perfusing myocardium and that perfusing the enteric circulation in fasting subjects has been confirmed with the use of the new analytical procedure. New data on coronary sinus and hepatic vein $\mathrm{A}-\mathrm{V}$ differences are listed in Table I. In addition to these sites, blood samples have been obtained from the greater saphenous vein immediately below its junction with the femoral vein, the vein having been exposed at this location for the introduction of a cardiac catheter. Large negative $\mathrm{A}-\mathrm{V}$ differences were encountered (Table II). Through the courtesy of Drs. Reuben Andres and Kenneth Zierler, twenty-two pairs of arterial and venous blood samples from the forearm muscles of fasting normal human subjects were made available. These samples were obtained by the techniques 
TABLE II

$A-V$ differences in UFA concentration from greater saphenous vein samples taken at cardiac catheterization *

\begin{tabular}{lccc}
\hline & \multicolumn{3}{c}{ UFA $(m E q . / L)}$. \\
\cline { 2 - 4 } Patient & Arterial & Venous & Diff. \\
\hline W. E. & .50 & .84 & -.34 \\
M. H. & .65 & .77 & -.12 \\
E. D. & .63 & 1.50 & -.87 \\
C. R. & .84 & 1.80 & -.96 \\
J. W. & .87 & 1.64 & -.77 \\
\hline
\end{tabular}

* The figures are means of duplicate analyses.

recently described (3), and were transported in the frozen state to this laboratory for analysis. In fifteen of these twenty-two pairs, there was found a positive arteriovenous difference in the UFA concentration which exceeded twice the standard error of the analytic procedure. Four of the remaining paired samples showed an equally significant negative difference, and the remainder were inconclusive. These data will be reported later in more detail and correlation with other arteriovenous differences. Finally, an attempt has been made to demonstrate extraction of UFA from blood perfusing the kidney in vivo. Suitable samples were obtained in nine patients undergoing cardiac catheterization. The arteriovenous differences ranged from +0.08 to $-0.03 \mathrm{mEq}$. per L., with both mean and median values of +0.01 , a difference which is unfortunately small in comparison with the experimental error.

There have been a number of instances in which it has been possible to administer glucose and insulin intravenously to a subject while obtaining sequential samples for the measurement of an arteriovenous difference of UFA concentration. One such example was reported previously (1), the venous site being the coronary sinus. Similar studies have since been carried out in three patients with catheters remaining in the hepatic vein, and in three in whom serial samples were obtained from the saphenous vein (Table III). The elapsed time between samplings was between thirty and forty-five minutes, and the doses of insulin and glucose were 0.1 unit per $\mathrm{Kg}$. body weight and $100 \mathrm{gm}$., respectively. In each experiment, the pre-existing arteriovenous difference was reduced to insignificance following the infusion of glucose and insulin.

Eight normal young men have been investigated with regard to the effects of nutritional state on UFA concentrations in blood drawn from antecubital veins. The subjects received no food after $6: 30$ P.M. on the day preceding the test period, and blood samples were drawn at about 9:00 A.M. and at hourly intervals thereafter until noon. The nutrients to be tested were fed immediately after the initial blood sample was drawn, each accompanied by approximately $300 \mathrm{ml}$. of water. On different days, the subjects received glucose, 50 gm., dl-alanine, 50 gm., 1-glutamic acid, 50 gm., and l-leucine, $25 \mathrm{gm}$. On another day, they fasted, receiving only the $300 \mathrm{ml}$. of water. The amino acids were found to be somewhat unpalatable in pure form, but were in general well tolerated. In three subjects there was mild gastric distress after alanine. The time course of the observed UFA concentrations is given in summary form in Figure 1. The difference between each measured UFA concentration and the baseline value at 9:00 A.M. has been determined for each subject, and comparable values averaged over all subjects. The figure indicates that the feeding of glucose or of any one of the amino acids reverses the normal upward trend of the plasma UFA concentration for a period of roughly two hours. It also appears that glucose is more effective in this respect than are the amino acids. These data have been submitted to statistical analysis, with the finding

TABLE III

\begin{tabular}{|c|c|c|c|c|c|}
\hline \multirow[b]{2}{*}{ atient } & \multirow{2}{*}{$\begin{array}{c}\text { Venous } \\
\text { site }\end{array}$} & \multirow{2}{*}{$\begin{array}{l}\text { Glucose } \\
\text { and } \\
\text { insulin }\end{array}$} & \multicolumn{3}{|c|}{ UFA $(m E q . / L)}$. \\
\hline & & & Arterial & Venous & Diff. \\
\hline B. & H.V. & $\begin{array}{l}\text { Before } \\
\text { After }\end{array}$ & $\begin{array}{l}.68 \\
.10\end{array}$ & $\begin{array}{l}.63 \\
.10\end{array}$ & $\begin{array}{l}.05 \\
.00\end{array}$ \\
\hline V. H. & H.V. & $\begin{array}{l}\text { Before } \\
\text { After }\end{array}$ & $\begin{array}{l}.89 \\
.25\end{array}$ & $\begin{array}{l}.78 \\
.25\end{array}$ & .11 \\
\hline . & H.V. & $\begin{array}{l}\text { Before } \\
\text { After }\end{array}$ & $\begin{array}{l}.42 \\
.08\end{array}$ & $\begin{array}{l}.31 \\
.08\end{array}$ & $\begin{array}{l}.11 \\
.00\end{array}$ \\
\hline E. & S.V. & $\begin{array}{l}\text { Before } \\
\text { After }\end{array}$ & $\begin{array}{l}.46 \\
.16\end{array}$ & $\begin{array}{r}1.09 \\
.19\end{array}$ & $\begin{array}{l}-.63 \\
-.03\end{array}$ \\
\hline S. & S.V. & $\begin{array}{l}\text { Before } \\
\text { After }\end{array}$ & $\begin{array}{l}.39 \\
.22\end{array}$ & $\begin{array}{l}.82 \\
.20\end{array}$ & $\begin{array}{r}-.43 \\
.02\end{array}$ \\
\hline S. & S.V. & $\begin{array}{l}\text { Before } \\
\text { After. }\end{array}$ & $\begin{array}{l}.36 \\
.13\end{array}$ & $\begin{array}{l}.50 \\
.12\end{array}$ & $\begin{array}{r}-.14 \\
.01\end{array}$ \\
\hline
\end{tabular}

* Samples from hepatic (H.V.) and saphenous (S.V.) veins. The figures are means of duplicate analyses. 
that the difference between the course of UFA concentrations after feeding any one of the nutriments and that observed during fasting is of significance ( $p$ less than 0.01). The greater effectiveness of glucose as compared with the amino acids is of borderline significance ( $p$ less than 0.05 for each comparison). ${ }^{2}$

\section{DISCUSSION}

With the use of a new analytical procedure which is superior in precision, specificity, and ease of performance, to the method formerly employed, it has been possible to confirm and extend earlier observations on the physiologic behavior of unesterified fatty acids in human blood plasma. In subjects undergoing cardiac catheterization, studies of arteriovenous differences leave no doubt that fatty acids in the form of soluble soaps are entering the circulation from the adipose tissue (this being the only tissue drained by the saphenous vein that could conceivably be the source of the UFA), and are being extracted from the blood by the myocardium and by the viscera whose collective venous drainage is available for sampling in the hepatic vein. The extraction in the viscera is in all probability greater than that indicated by the measured $\mathrm{A}-\mathrm{V}$ difference, because omental fat may be expected to be adding UFA to the blood within this enteric circulation at the same time that other tissues are extracting it. That skeletal muscle may also extract UFA is almost as certain on the basis of the measured $\mathrm{A}-\mathrm{V}$ differences in the samples submitted by Drs. Andres and Zierler. Their preparation of human forearm does not permit certain knowledge that no venous drainage from adipose tissue is included in the samples collected, and the addition of UFA to the blood by adipose tissue in a quantity exceeding that being simultaneously extracted by muscles is believed to be the explanation of the small number of negative $\mathrm{A}-\mathrm{V}$ differences encountered. The attempt to demonstrate $\mathrm{A}-\mathrm{V}$ differences across the kidney was perhaps foredoomed to failure, as the renal blood flow is so large relative to metabolic demands. From the average renal A-V oxygen difference noted by Cargill and Hickam (4), and using the same assumptions as in previous calculations, it is found that if kidney were to obtain all of its energy by the oxidation of unesterified

\footnotetext{
2 The author is indebted to Mr. Felix Moore, Biometrics Research Section, National Heart Institute, for guidance in this analysis.
}

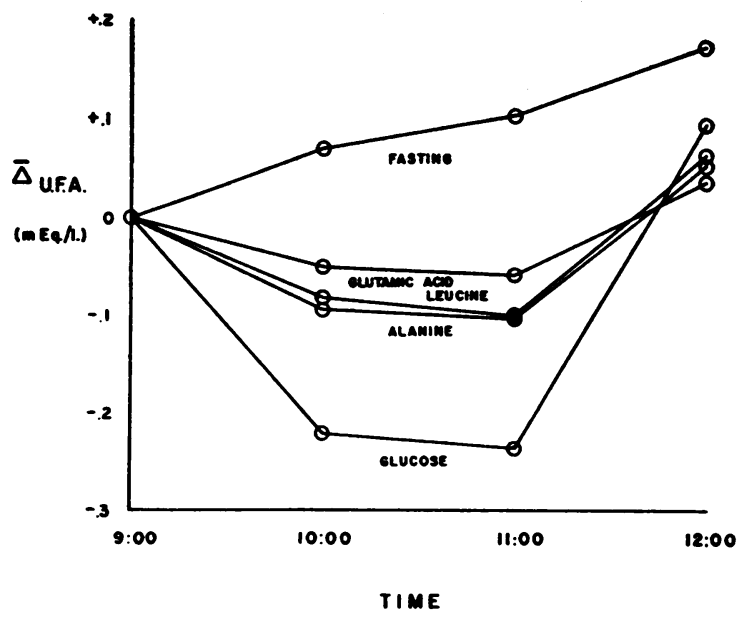

Fig. 1. Effect of Feeding on Unesterified Fatty Acid

The average change in UFA concentration in the antecubital vein, the reference being the basal value at 9:00 A.M., is plotted as a function of time. All feedings were given immediately after the initial blood sample was obtained.

fatty acids, an $\mathrm{A}-\mathrm{V}$ difference of only $0.05 \mathrm{mEq}$. per L. would be expected. The values encountered are consonant with the belief that unesterified fatty acid as a substrate is available to renal tissue, but the present data are insufficient to prove that extraction occurs at all, and certainly permit no quantitative conclusions.

A matter of importance is the finding that after administration of glucose and insulin to human subjects, there occurs, coincident with a decreased arterial level of UFA, complete cessation of net transport of UFA as indicated by the abolition of $\mathrm{A}-\mathrm{V}$ differences. The events observed indicate that the transport of fat as UFA is controlled primarily by the adipose tissue, and that when glucose is made available, there is first a decrease in the liberation of UFA from depot fat, a resultant drop in arterial UFA concentration, and then a cessation of extraction by other tissues. The reverse sequence is not reasonable, as in that case the arterial level would be expected to rise as tissue extraction was halted. A similar direct relationship between arterial level of substrate and the tissue extraction has been observed by Bing, Siegel, Vitale, Balboni, Sparks, Taeschler, Klapper, and Edwards (5) in the case of the non-lipid substrates that they have studied extensively in the human myocardium in vivo. In the present studies the combination of glucose and insulin has been employed in order to minimize the time that the subject must spend with the catheter in place. 
It seems unlikely that any major difference exists between the results encountered with this procedure and those that would result more slowly from the administration of glucose alone, so long as the subject has a normally functioning pancreas.

Although direct experimental proof is lacking, it seems reasonable to speculate that the ingestion of amino acids, which, like the ingestion of glucose, causes a reduction in the circulating UFA concentration, will result in a diminution or abolition of $\mathrm{A}-\mathrm{V}$ differences as well, implying a primary decrease in liberation of UFA from the adipose tissue. The mechanism of control of this process is as yet completely unknown. The mechanism, whatever its nature, appears to be activated by some receptor system capable of detecting the availability of oxidizable, energy-yielding substrates even though these differ metabolically as much as do glucose, alanine, glutamic acid, and leucine. The last is of special interest, as a representative of the ketogenic amino acids, whose intermediary metabolism is believed to have nothing in common with the metabolism of glucose until degradation has proceeded to the level of acetate. The availability of calories from non-fat sources appears to be so far the chief determinant of plasma UFA concentrations; any operation making calories more available results in a decrease, whereas the opposite causes an increase. The exception appears to be the response to epinephrine, which has been shown $(1,2)$ to produce an immediate rise in UFA levels. The effect of epinephrine is of particular interest in view of the older work, cited by Wertheimer and Shapiro in their excellent review (6), related to the possibility of nervous control of the function of adipose tissue.

Consideration of the present data leads to two inferences which are being tested in current experimental work. First is the almost inevitable conclusion, derived from the measured $\mathrm{A}-\mathrm{V}$ differences, that the turnover time of an isotopic label in the plasma UFA fraction should be extremely short. Second is the expectation of elevated plasma UFA concentrations in diabetic patients who are not under insulin control, a clinical situation in which non-fat calories are less than normally available. It is of interest that Dangerfield and Smith (7) some time ago reported on changes in the electrophoretic pattern of plasma taken from acute diabetic acidosis, changes which re- semble closely those produced by the addition of sodium oleate to serum in vitro (8).

Another reasonable inference to be drawn from these data, but concerning which there are as yet no direct experimental measurements, is that the fatty acids in the unesterified fraction, insofar as they are derived from the adipose tissue, may be expected to have the same chemical characteristics as regards chain length and unsaturation as do the fatty acids of the depot fat. The depots of higher mammals are, in general, made up primarily of the glycerides of stearic, palmitic, and oleic acids. The assumption of an average molecular weight of 275 for the unesterified fatty acids of blood plasma is based on this inference, and if it should be proved incorrect, the values of oxygen extraction ratio given in this and the preceding paper will require appropriate revision.

\section{SUMMARY}

Employing a newly developed analytical procedure for the determination of the concentration of unesterified fatty acid (UFA) in blood plasma, it has been possible to demonstrate in fasting human subjects arteriovenous differences indicative of a net transport of fatty acid from adipose tissue to myocardium, skeletal muscle, and viscera. The administration of glucose and insulin causes a sharp decrease in UFA concentrations and an abolition of the arteriovenous differences. Alanine, leucine, and glutamic acid administered by mouth to fasting humans have an effect like that of glucose in reducing UFA concentrations, though the decreases are less dramatic. It is suggested that adipose tissue responds, through some mechanism sensitive to the availability of non-fat calories, by liberating more or less UFA into the blood and so maintaining a "caloric homeostasis."

\section{ACKNOWLEDGMENT}

The assistance of the various staff members of the National Institutes of Health who made patients or blood samples available to the author is gratefully acknowledged.

\section{REFERENCES}

1. Gordon, R. S., Jr., and Cherkes, A., Unesterified fatty acid in human blood plasma. J. Clin. Invest., 1956, 35, 206.

2. Dole, V. P., A relation between non-esterified fatty 
acids in plasma and the metabolism of glucose. J. Clin. Invest., 1956, 35, 150.

3. Andres, R., Cader, G., and Zierler, K. L., The quantitatively minor role of carbohydrate in oxidative metabolism by skeletal muscle in intact man in the basal state. Measurements of oxygen and glucose uptake and carbon dioxide and lactate production in the forearm. J. Clin. Invest., 1956, 35, 671.

4. Cargill, W. H., and Hickam, J. B., The oxygen consumption of the normal and the diseased human kidney. J. Clin. Invest., 1949, 28, 526.

5. Bing, R. J., Siegel, A., Vitale, A., Balboni, F., Sparks,
E., Taeschler, M., Klapper, M., and Edwards, S., Metabolic studies on the human heart in vivo. I. Studies on carbohydrate metabolism of the human heart. Am. J. Med., 1953, 15, 284.

6. Wertheimer, E., and Shapiro, B., The physiology of adipose tissue. Physiol. Rev., 1948, 28, 451.

7. Dangerfield, W. G., and Smith, E. B., Electrophoretic examination of the effect of heparin on serum lipoproteins. Biochem. J., 1955, 59, vi.

8. Gordon, R. S., Jr., Interaction between oleate and the lipoproteins of human serum. J. Clin. Invest., 1955, 34, 477. 